Elisabeth C. Oliveira Santos 1

\title{
Avaliação dos níveis de exposição ao mercúrio entre índios Pakaanóva, Amazônia, Brasil
}

\author{
Mercury exposure among Pakaanóva \\ Indians, Amazon Region, Brazil
}

Volney de Magalhães Câmara ${ }^{2}$

Edilson da Silva Brabo ${ }^{1}$

Edvaldo Carlos Brito Loureiro 1

Iracina Maura de Jesus 1

Kleber Fayal 1

Fernanda Sagica 1

\footnotetext{
1 Seção de Meio Ambiente, Instituto Evandro Chagas, Fundação Nacional de Saúde. Av. Almirante Barroso 492, Belém, PA 66090-000, Brasil.

2 Núcleo de Estudos de Saúde Coletiva, Centro de Ciências da Saúde, Universidade Federal do Rio de Janeiro. Av. Brigadeiro Trompowsky $s / n$, Hospital Universitário, Ala Sul, Rio de Janeiro, 21941-530, Rio de Janeiro, Brasil.
}

\begin{abstract}
A cross-sectional study was conducted to evaluate mercury (Hg) exposure among 910 Pakaanóva Indians from the counties of Guajará Mirim and Nova Marmoré, Rondônia State, Brazil. Individual hair samples were taken from the occipital region, and Hg was measured by atomic absorption spectrometry with cold vapor generation. Mean Hg in hair samples was 8.37 $\mu \mathrm{g} / \mathrm{g}$ (range 0.52-83.89), indicating high exposure. Young children (<2 years old) showed a mean $\mathrm{Hg}$ of $10.54 \mu \mathrm{g} / \mathrm{g}$, and children from 3 to 5 years old had a mean $\mathrm{Hg}$ of $9.34 \mu \mathrm{g} / \mathrm{g}$. Mercury levels in women $(8,91 \mu \mathrm{g} / \mathrm{g})$ were higher than in men $(7.55 \mu \mathrm{g} / \mathrm{g})$, and this difference was significant $(t=$ 3.26; $p<0.01)$. These results indicate the need for surveillance programs and complementary studies including the Pakaanóva Indians in Rondônia State.
\end{abstract}

Key words Mercury Poisoning; Mercury; Mining; South American Indians

Resumo Um estudo seccional foi realizado para avaliar os níveis de exposição ao mercúrio $(H g)$ entre 910 índios Pakaanóva, residentes nos municípios de Guajará Mirim e Nova Marmoré no Estado de Rondônia, Brasil. Amostras de cabelo da região occipital foram coletadas de cada participante do estudo e os teores de Hg determinados por Espectrofotometria de Absorção Atômica com Geração de Vapor Frio. Os teores médios de $\mathrm{Hg}$ nas amostras de cabelo foram de 8,37 $\mathrm{g} / \mathrm{g}$ $(0,52-83,89 \mu \mathrm{g} / \mathrm{g})$, indicando exposição elevada. Crianças de até 2 anos e entre 3 e 5 anos de idade

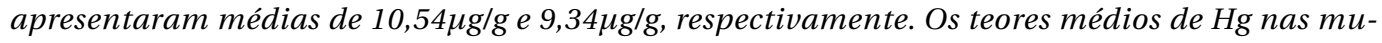
lheres $(8,91 \mu \mathrm{g} / \mathrm{g})$ são mais elevados do que os valores observados nos homens $(7,55 \mu \mathrm{g} / \mathrm{g})$, sendo esta diferença estatisticamente significante $(t=3,26 ; p<0,01)$. Estes resultados indicam a necessidade de desenvolvimento de programas de vigilância e estudos complementares envolvendo os índios Pakaanóva no Estado de Rondônia.

Palavras-chave Intoxicação por Mercúrio; Mercúrio; Mineração; Índios Sul-Americanos 


\section{Introdução}

As transformações sociais e econômicas que ocorreram na Amazônia brasileira nas últimas décadas contribuíram para modificações no perfil de morbidade das populações indígenas desta região. Projetos de desenvolvimento, movimentos migratórios e o rápido e abrupto contato com a sociedade "civilizada" estão relacionados com doenças infecciosas e parasitárias (Conklin, 1994; Santos \& Coimbra Jr., 1994). A partir do final da década de 70 , atividades de mineração de ouro contribuíram também para elevadas emissões de mercúrio no ambiente. Nos processos de produção de ouro o mercúrio sob a forma metálica é utilizado para formar um amálgama para recuperação do ouro de granulação fina. Em seguida, este amálgama é queimado, via de regra ao ar livre, e o mercúrio liberado para a atmosfera na forma de vapor, restando o ouro. O mercúrio metálico pode, posteriormente, ser depositado nos rios e, por intermédio da cadeia biológica, contaminar os peixes na sua forma mais tóxica: o metilmercúrio (Câmara \& Corey, 1992; Santos et al., 1995).

Nesta situação, entre os diversos grupos populacionais que estão expostos, destacam-se os índios. O peixe é a principal fonte de proteínas das comunidades indígenas da Amazônia. Brabo et al. (1999), em estudo realizado no Estado do Pará, observaram elevado consumo de peixe e falta de outras alternativas como fonte de alimentação entre os índios. Dentre os peixes carnívoros e não carnívoros mais consumidos foram citados tucunaré, pacu, jaraqui, traíra, aracu, matrinchã e caratinga. Em um estudo seccional para avaliar exposição por mercúrio entre 330 índios Mundurukú da comunidade denominada Sai Cinza no Estado do Pará, Santos et al. (2001) encontraram grande consumo de peixe que foi associado com teores de mercúrio no tecido capilar, sendo $14,45 \mu \mathrm{g} / \mathrm{g}$ para crianças entre 7 a 12 anos de idade, $15,70 \mu \mathrm{g} / \mathrm{g}$ para mulheres entre 14 e 44 anos e $14,1 \mu \mathrm{g} / \mathrm{g}$ para os outros indivíduos. Barbosa et al. (1998), em pesquisa envolvendo 251 mulheres e crianças indígenas que foram selecionadas ao longo do rio Madeira e da Reserva Kayapó, mostraram entre as mulheres uma média de mercúrio

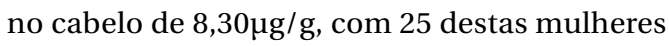
apresentando teores acima de 10,0 $\mathrm{g} / \mathrm{g}$. Também a correlação entre o tempo de amamentação e a concentração de mercúrio no tecido capilar foi positiva $(\mathrm{r}=0,51$ e $\mathrm{p}=0,02)$ para as crianças indígenas.

Na Amazônia da Guiana Francesa, Frery et al. (2001) realizaram um estudo que apontou
57\% dos 235 habitantes da comunidade Wayána com teores de mercúrio no cabelo acima de $10,0 \mu \mathrm{g} / \mathrm{g}$ e 270 amostras de peixes da região apresentaram teores de mercúrio total em músculos de até $1,62 \mathrm{mg} / \mathrm{kg}$. Os autores concluíram que a exposição ao mercúrio nesta população estava relacionada com o elevado consumo de peixes.

A exposição ao metilmercúrio em comunidades indígenas associada ao consumo de peixes não ocorre somente em países amazônicos. Entre 89 índios norte americanos da tribo Ojibwa, $91,4 \%$ relataram que consumiam peixes, embora os teores de mercúrio em cabelos de todas as amostras analisadas apresentassem valores abaixo de 3,0 $\mu \mathrm{g} / \mathrm{g}$ (Gerstenberger et al., 1997). Em outro estudo de 327 índios Chippewa, o consumo de peixes foi elevado e 205 das amostras de sangue apresentaram concentrações de $\mathrm{Hg}$ acima de 5,0 $\mu \mathrm{g} / \mathrm{L}$. Foi observada a existência de elevada correlação entre o consumo de peixe com o aumento dos teores de $\mathrm{Hg}$ no sangue (Peterson et al., 1994).

\section{Material e métodos}

Um estudo seccional para avaliar os níveis de exposição ao mercúrio foi realizado entre os índios Pakaanóva, residentes nos municípios de Guajará Mirim e Nova Marmoré no Estado de Rondônia, área sob a influência da atividade garimpeira da bacia do Rio Madeira (Figura 1). A população indígena estimada nesses municípios é de 1.930 indivíduos (Instituto Socioambiental, 1997). Neste estudo, participaram 910 índios distribuídos entre as aldeias de Deolinda $(n=53)$, Lage $(n=108)$, Ricardo Franco $(n=114)$, Rio Negro Ocaia $(n=154)$, Ribeirão $(n=84)$, Sagarana $(n=39)$, Sotério $(n=117)$, Santo André ( $\mathrm{n}=114)$ e Tanajura ( $\mathrm{n}=127)$, localizadas a diferentes distâncias de Guajará Mirim. Outros indivíduos não participaram do estudo em função de estarem ausentes do local, serem população não indígena ou recusas. Em geral, as condições de vida e saúde são precárias nas localidades. A água consumida é obtida dos rios próximos e sem nenhum tratamento. A principal atividade ocupacional é a agricultura (149) e existem ainda 105 estudantes. Os procedimentos de campo incluíram uma visita prévia para contatos e divulgação da importância do estudo.

As amostras de tecido capilar foram coletadas da região occipital cortadas até um centímetro do escalpo. Cada mecha continha em média cerca de 100 fios de cabelo que foram acondicionadas em envelopes brancos devida- 


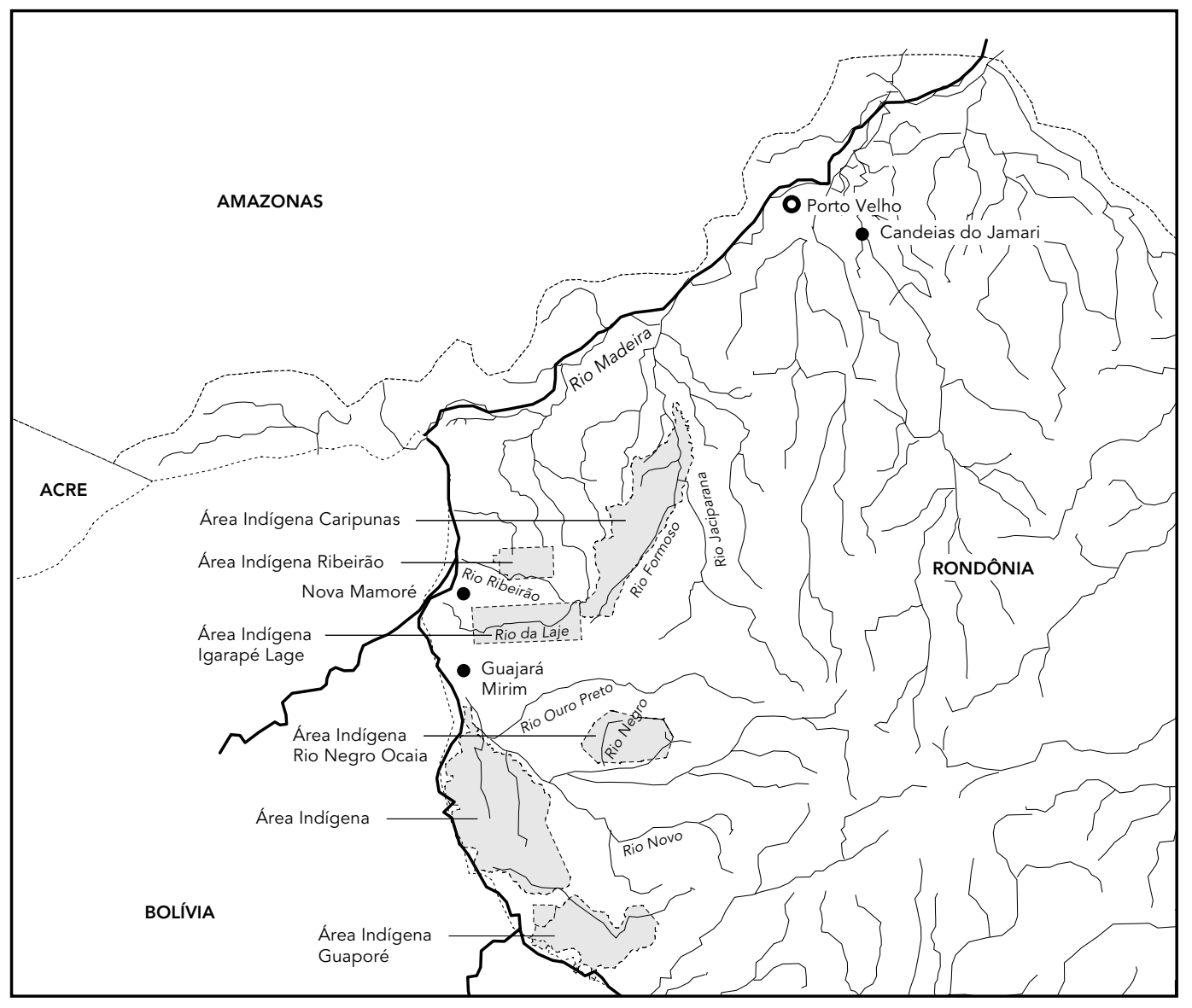

municípios

reservas indígenas

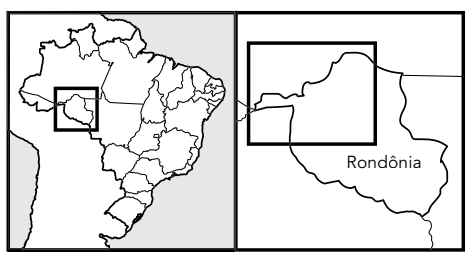

mente identificados e armazenados em temperatura ambiente num local adequado para transporte até o laboratório. Os procedimentos analíticos para determinação de mercúrio total foram realizados na Seção de Meio Ambiente do Instituto Evandro Chagas de acordo com o método proposto por Akagi et al. (1996). Foi utilizado um Espectrofotômetro de Absorção Atômica com sistema de geração de vapor frio acoplado, Modelo Mercury Analyzer Hg-
3500. A qualidade analítica dos resultados é avaliada pela determinação de $\mathrm{Hg}$ em amostras certificadas e exercícios de intercalibração laboratorial.

Os dados obtidos foram armazenados no programa dBase versão 3.0, e o tratamento estatístico pelos programas Stata 5.0 e BioEstat 2.0 .

Este estudo foi realizado atendendo aos preceitos éticos da Resolução 196/96 do Conselho 
Nacional de Saúde e Declaração de Helsinki Tóquio e com aprovação do Comitê de Ética em Pesquisa do Instituto Evandro Chagas.

\section{Resultados}

A distribuição por sexo mostrou que a população estudada estava composta por 549 mulheres $(60,3 \%)$ e 361 homens $(39,7 \%)$ com idades variando entre 6 meses e 90 anos. A média de mercúrio total no tecido capilar dos 910 indivíduos que participaram do estudo foi de 8,37 $\mu \mathrm{g} / \mathrm{g}$, com amplitude entre 0,52 e $83,89 \mu \mathrm{g} / \mathrm{g}$. Na Tabela 1, observa-se que a distribuição de Hg em relação à mediana $(6,89 \mu \mathrm{g} / \mathrm{g})$ mostrouse próxima ao Limite de Tolerância Biológico (LTB) de 6,0 $\mu \mathrm{g} / \mathrm{g}$, adotado pela Organização Mundial de Saúde, (WHO, 1990), verificandose significância estatística em relação aos níveis encontrados acima do valor da mediana (Qui quadrado $=474,15 \mathrm{GL}=1$ e $\mathrm{p}<0,01$ ).

Os valores médios de Hg mais elevados foram observados em crianças com idades menores que 5 anos, com destaque para aquelas situadas na faixa etária entre 0 e 2 anos (média de $10,5 \mu \mathrm{g} / \mathrm{g}$ ) e 3 a 5 anos com média de $9,3 \mu \mathrm{g} / \mathrm{g}$.

As análises mostraram que os teores médios de Hg nas mulheres são mais elevados do que os valores observados nos homens, sendo de $8,91 \mu \mathrm{g} / \mathrm{g}$ e $7,55 \mu \mathrm{g} / \mathrm{g}$, respectivamente. Mediante o teste t de Student, observou-se que esta diferença é estatisticamente significante $(\mathrm{t}=$ $3,26 ; \mathrm{p}<0,001)$. A avaliação da correlação linear entre idade e os teores de Hg no tecido capilar foi positiva e não significante com $r=$ 0,0043 e $p>0,05$.
A Figura 1 mostra a distribuição das concentrações de Hg no tecido capilar na população dividida em 3 grupos distintos, cujo critério de escolha considerou os possíveis impactos que a exposição ao $\mathrm{Hg}$ pode acarretar ao desenvolvimento neuropsicomotor das crianças e à saúde de mães e conceptos. O primeiro grupo é representado por crianças com idade até 12 anos; o segundo, por mulheres no período fértil, com idade variando entre 14 e 44 anos; e o terceiro, por outros indivíduos que não se enquadravam nas categorias anteriores. Os teores médios de Hg não apresentaram variações significativas. Os valores de $\mathrm{Hg}$ foram da ordem de $7,93 \mu \mathrm{g} / \mathrm{g}$ para o primeiro grupo; $8,55 \mu \mathrm{g} / \mathrm{g}$ para o segundo; e $8,42 \mu \mathrm{g} / \mathrm{g}$ para os indivíduos do terceiro grupo. Observou-se ainda que, no primeiro grupo, $75 \%$ das crianças apresentaram teores de até $10,42 \mu \mathrm{g} / \mathrm{g}$. Os demais $25 \%$ mostraram níveis que alcançaram $83,8 \mu \mathrm{g} / \mathrm{g}$, que foram os mais elevados, destacados como pontos anômalos na Figura 2.

No grupo das mulheres em idade reprodutiva, os teores de $\mathrm{Hg}$ em $75 \%$ das participantes não ultrapassaram $10,88 \mu \mathrm{g} / \mathrm{g}$, apresentando um comportamento similar ao observado no grupo anterior, enquanto os $25 \%$ remanescentes encontraram-se acima desses níveis, tendo sido de 39,4 $\mu \mathrm{g} / \mathrm{g}$ o valor mais elevado.

O terceiro grupo apresentou um comportamento semelhante aos descritos anteriormente, em relação ao terceiro quartil $(10,77 \mu \mathrm{g} / \mathrm{g})$, com destaque para o valor anômalo de $27,5 \mu \mathrm{g} / \mathrm{g}$.

Em relação ao desenvolvimento de atividades laborais e os níveis de $\mathrm{Hg}$, foi verificado que índios que trabalhavam na agricultura apresentaram mediana de $7,45 \mu \mathrm{g} / \mathrm{g}$ e o grupo

Tabela 1

Concentração de mercúrio total em tecido capilar $(\mu \mathrm{g} / \mathrm{g})$ por faixa etária de 910 índios Pakaánova.

Rondônia, Brasil, 1997

\begin{tabular}{lccccc}
\hline Faixa Etária & $\mathbf{n}$ & $\begin{array}{c}\text { Concentração } \\
\text { de Hg em tecido } \\
\text { capilar }(\boldsymbol{\mu g} / \mathbf{g})\end{array}$ & Mediana & Amplitude & Desvio Padrão \\
\hline $0-2$ & 57 & 10,54 & 7,20 & $1,48-83,89$ & 13,06 \\
$3-5$ & 115 & 9,34 & 7,33 & $1,67-47,22$ & 6,86 \\
$6-10$ & 152 & 8,16 & 6,68 & $0,52-63,81$ & 6,93 \\
$11-15$ & 114 & 6,86 & 5,94 & $0,65-31,11$ & 4,39 \\
$16-25$ & 177 & 8,45 & 7,06 & $0,65-39,42$ & 5,55 \\
$26-35$ & 114 & 8,56 & 7,30 & $1,37-28,64$ & 5,00 \\
$36-45$ & 50 & 8,39 & 7,96 & $1,49-21,25$ & 5,01 \\
$>45$ & 131 & 7,84 & 6,77 & $1,37-25,84$ & 4,49 \\
Total & 910 & 8,37 & 6,89 & $0,52-83,89$ & 6,44 \\
\hline
\end{tabular}


formado por indivíduos que não referiram suas atividades ocupacionais mostraram teores mais altos $(8,87 \mu \mathrm{g} / \mathrm{g})$.

A distribuição de Hg no tecido capilar entre os subgrupos estudados, utilizando a mediana como medida de tendência central é mostrada na Tabela 2. Nota-se que os valores mais elevados foram verificados nas populações que habitam às margens dos rios Pacaá-Nova e Guaporé, com destaque para a comunidade de Rio Negro Ocaia com valores da ordem de 13,07 $\mu \mathrm{g} / \mathrm{g}$. Ao contrário, nos grupos que não estão localizados às margens dos rios (Lage e Ribeirão) os valores de $\mathrm{Hg}$ foram de 3,14 e 3,49 $\mathrm{g} / \mathrm{g}$, respectivamente. Este fato sugere que os hábitos alimentares, sobretudo o consumo elevado de peixe, sejam responsáveis pelo atual quadro de exposição ao Hg dessas comunidades indígenas.

A Figura 3 compara os teores médios de mercúrio no tecido capilar, distribuídos por faixa etária, entre a população dos Pakaanóva, a comunidade indígena Mundurukú (Posto Indígena Sai Cinza) e duas populações ribeirinhas (São Luís do Tapajós e Brasília Legal) no Estado do Pará, todas situadas às margens do rio Tapajós, numa área sob influência da atividade garimpeira, considerada de risco. O comportamento dos níveis de $\mathrm{Hg}$ entre as duas comunidades indígenas é bastante semelhante, com destaque para a uniformidade constante dos teores de $\mathrm{Hg}$ por faixa etária. Ao contrário do que ocorre com as comunidades de São Luís do Tapajós e Brasília Legal onde os valores de $\mathrm{Hg}$ decrescem com o aumento das faixas etárias.

\section{Discussão e conclusão}

A área habitada pelos Pakaanóva está sob a influência da Bacia do Rio Madeira, onde a atividade garimpeira é praticada e ocorre liberação de Hg para o ambiente. Rondônia é um Estado em que as queimadas representam uma importante fonte de emissão de mercúrio para a atmosfera, mediante a queima da biomassa contendo Hg. O desmatamento também con-

Figura 2

Mercúrio total em cabelos $(\mu \mathrm{g} / \mathrm{g})$ por grupos selecionados dos Índios Pakaánova. Rondônia, Brasil, 1997.

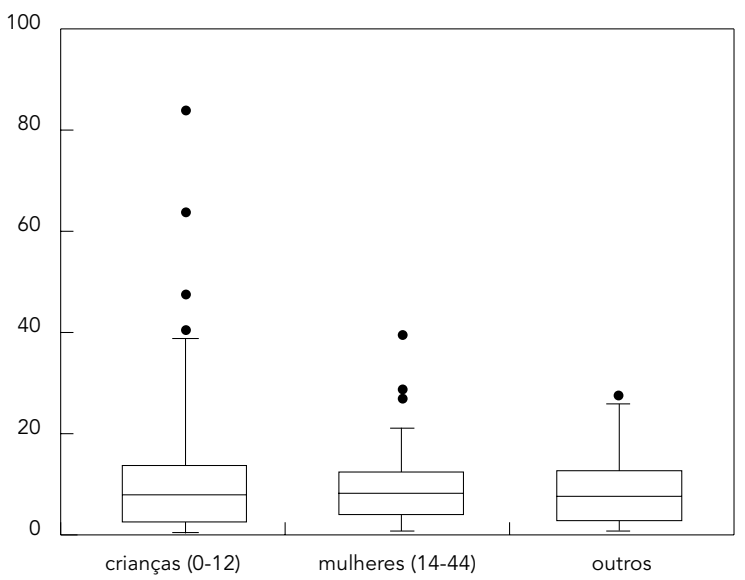

Tabela 2

Distribuição dos teores de Hg no tecido capilar dos subgrupos dos Índios Pakaánova. Rondônia, Brasil, 1997.

\begin{tabular}{|c|c|c|c|c|c|}
\hline \multirow[t]{2}{*}{ Localidades } & \multirow[t]{2}{*}{ Indivíduos } & \multirow[t]{2}{*}{$\%$} & \multicolumn{3}{|c|}{$\begin{array}{l}\text { Concentração } \\
\text { de } \mathrm{Hg}(\mu \mathrm{g} / \mathrm{g})\end{array}$} \\
\hline & & & Inferior & Mediana & Superior \\
\hline Deolinda & 53 & 5,8 & 1,04 & 4,85 & 83,89 \\
\hline Lage & 108 & 11,9 & 0,52 & 3,14 & 63,81 \\
\hline Ricardo Franco & 114 & 12,5 & 3,04 & 6,81 & 47,74 \\
\hline Rio Negro Ocaia & 154 & 16,9 & 3,26 & 13,07 & 31,96 \\
\hline Ribeirão & 84 & 9,2 & 0,65 & 3,49 & 24,93 \\
\hline Sagarana & 39 & 4,3 & 2,28 & 6,24 & 13,21 \\
\hline Soterio & 117 & 12,9 & 2,18 & 5,79 & 31,11 \\
\hline Santo André & 114 & 12,5 & 1,93 & 9,14 & 19,73 \\
\hline Tanajura & 127 & 14,0 & 1,54 & 9,14 & 40,35 \\
\hline Total & 910 & 100 & 0,52 & 6,89 & 83,89 \\
\hline
\end{tabular}


Figura 3

Teores de mercúrio total em cabelos em $\mu \mathrm{g} / \mathrm{g}$ por localidades dos Índios Pakaánova. Rondônia, Brasil, 1997.

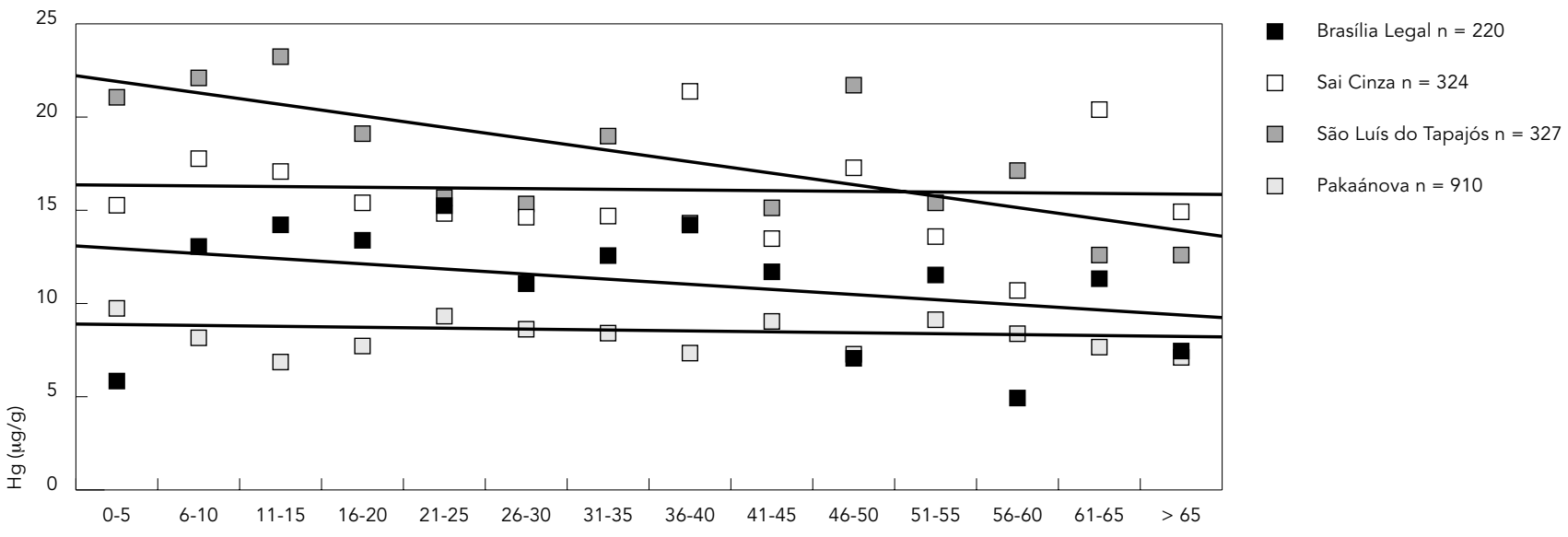

tribui para a mobilização do mercúrio dos solos para os ecossistemas aquáticos pela lixiviação do Hg (Roulet et al., 1998; Veiga et al., 1994).

A população estudada apresenta teores de mercúrio no cabelo que variam de $0,52 \mu \mathrm{g} / \mathrm{g}$ até $83,89 \mu \mathrm{g} / \mathrm{g}$, numa distribuição irregular por idade e sexo; porém, homogênea no tocante ao risco da exposição ao metal, cujo veículo é o pescado, ficando esta distribuição por conta das peculiaridades culturais, no que diz respeito à freqüência de ingestão e espécies consumidas de pescado.

Pode-se considerar que a exposição desta população indígena é elevada, tendo em vista que a Organização Mundial da Saúde cita $2 \mu \mathrm{g} / \mathrm{g}$ para populações não expostas e $6 \mu \mathrm{g} / \mathrm{g}$ como indicador de exposição (WHO, 1990). Os números encontrados indicam níveis de exposição ao Hg semelhantes aos encontrados por Barbosa et al. (1998) para as mulheres residentes na Reserva Kayapó $(8,30 \mu \mathrm{g} / \mathrm{g})$ no Sul do Pará e inferiores aos obtidos por Santos et al. (2001) para os índios Mundurukús estudados $(16,0 \mu \mathrm{g} / \mathrm{g})$ no Estado do Pará.

Torna-se relevante também o fato de crianças estarem expostas a concentrações elevadas de mercúrio, uma vez que estão em uma situação de maior risco para efeitos adversos à saúde, por encontrarem-se em fase de desenvolvimento neuropsicomotor. Além disso, quando essas crianças apresentam níveis de exposição ao mercúrio semelhantes ou maiores que os adultos, pode-se estimar que os riscos para elas são mais elevados em virtude de sua menor massa corporal (Asmus et al., 1996).

Comparando os teores de Hg de São Luís do Tapajós e Brasília Legal, comunidades não índias situadas em áreas consideradas como de risco de contaminação por $\mathrm{Hg}$ da atividade garimpeira, chama a atenção a tendência diferente das curvas constatadas entre comunidades indígenas e não indígenas. Quando se distribuem os teores médios de mercúrio medidos em cabelo pelas diferentes faixas etárias em populações não índias, a Figura 2 oferece no primeiro momento teores mais elevados na população mais jovem, e um acentuado decréscimo em direção a idades mais adultas da amostra, o que parece incoerente quando se analisa um agente de natureza cumulativa no organismo. É válido pensar que, ao longo dos últimos 30 anos, as crianças que antes nasciam sem a exposição ao mercúrio, hoje recebem, já na vida intra-uterina, teores cada vez mais relevantes do metal, que são acumulados durante a vida adulta, com base em um patamar pré existente (Santos, 2001; Santos et al., 2000). Nas comunidades indígenas, a relação dos teores de $\mathrm{Hg}$ com a idade é quase uma constante. O que muda? Muda a freqüência com que uma população índia consome pescado e é exposta ao risco de acumulação, o tipo de pescado e as quantidades consumidas, maiores, dentro de uma dieta alimentar menos variada.

Neste sentido, dada a dependência do peixe como fonte de alimentação das comunidades indígenas e a associação deste padrão ali- 
mentar com o aumento da exposição ao mercúrio, é importante recomendar a inserção destas questões em programas de vigilância ambiental em saúde, que considerem as peculiaridades dos sistemas de nutrição e saúde dos Pakaanóva, bem como novas pesquisas para avaliar os processos de acumulação mercurial destas populações.

\section{Agradecimentos}

Os autores agradecem às instituições Fundação Nacional de Saúde e Fundação Nacional do Índio - Rondônia e aos enfermeiros Aldo Pituaka e Sílvia Cavalcante pelo apoio ao trabalho de campo, bem como às seguintes pessoas pelo suporte técnico às atividades: Raimundo Pio Girard Martins, Airton de Jesus das Graças Teixeira, Raimundo Otávio Paixão, José Góes dos Santos, Vanda do Socorro da Costa Siqueira, Edna Cabral Trindade, Maria América Silva Guimarães e Maria de Nazaré Fernandes.

\section{Referências}

AKAGI, H.; MALM, O. \& BRANCHES, F. J. P., 1996. Human exposure to mercury due to mining in the Amazon, Brazil - A review. Environmental Sciences, 3:199-211.

ASMUS, C. F.; RUZANNY, M. H.; BARKER, R. S. L. \& MEIRELLES, Z. V., 1996. Riscos ocupacionais na infância e adolescência - Uma revisão. Jornal de Pediatria, 72:203-208.

BARBOSA, A. C.; SILVA S. R. \& DOREA, J. G., 1998. Concentration of mercury in hair of indigenous mothers and infants from the Amazon basin. Archives of Environmental Contamination Toxicology, 34:100-105.

BRABO, E. S.; SANTOS, E. C. O.; JESUS, I. M.; MAS CARENHAS, A. F. \& FAIAL, K. F., 1999. Níveis de mercúrio em peixes consumidos pela comunidade indígena de Sai Cinza na Reserva Munduruku, Município de Jacareacanga, Estado do Pará, Brasil. Cadernos de Saúde Pública, 15:325-331.

CÂMARA, V. M. \& COREY, G., 1992. Epidemiologia e Meio Ambiente. O Caso dos Garimpos de Ouro no Brasil. México, DF: Centro Pan-Americano de Ecología Humana y Salud, Organización Panamericana de Salud. 
CONKLIN, B. A., 1994. O sistema médico Wari (Pacaás Novos). In: Saúde e Povos Indígenas (R. V. Santos \& C. E. A. Coimbra Jr., org.), pp. 161-186, Rio de Janeiro: Editora Fiocruz.

FRERY, N.; MAURY-BRACHET, R.; MAILLOT, E.; DEHEEGER, M.; MERONA, B. \& BOUDOU, A., 2001. Gold mining activities and mercury contamination of native ameridian communities in French Guiana: Key role of fish in dietary uptake. Environmental Health Perspectives, 109:449-456.

GERSTENBERGER, S. L.; TAVRIS, D. R.; HANSEN, L. K.; PRATT-SHELLEY, J. \& DELLINGER, J. A., 1997. Concentrations blood and hair mercury and serum PCBs in an Ojibwa population that consumes Great Lakes region fish. Journal of Toxicology-Clinical Toxicology, 35:377-386.

INSTITUTO SOCIOAMBIENTAL, 1997. Povos Indigenas no Brasil: Wari. 21 Maio 2002 <http://www.socio ambiental.org/website/pib/epi/wari/wari.htm>.

PETERSON, D. E.; KANAREK, M. S.; KUYKENDALL, M. A.; DIEDRICH, J. M.; ANDERSON, H. A.; REMINGTON, P. L. \& SHEFFY, T. B., 1994. Fish consumption patterns and blood mercury levels in Wisconsin Chippewa indians. Archives of Environmental Health, 49:53-58.

ROULET, M.; LUCCOTTE, M.; SAINT-AUBIN, A.; RHEAULT, I.; FARELA, N.; JESUS-SILVA, E.; DEZENCOURT, J.; SOUSA-PASSOS, C. J.; SANTOS-SOARES, G.; GUIMARÃES, J. R.; MERGLER, D. \& AMORIM, M., 1998. The geochemistry of mercury in central Amazonian soils developed on the Alter do Chão formation of the lower Tapajós river valley, Pará state, Brazil. Science of the Total Environment, 223:1-24.

SANTOS, R. V. \& COIMBRA Jr., C. E. A., 1994. Contato, mudanças sócio-econômicas e bioantropologia dos Tupi-Mondé da Amazônia brasileira. In: Saúde e Povos Indígenas (R. V. Santos \& C. E. A. Coimbra Jr., org.), pp. 189-212, Rio de Janeiro: Editora Fiocruz.
SANTOS, E. C. O., 2001. Enquêtes sanitaires et contaminations mercurielles en Amazonie brésilienne. In: Le Mercure en Amazonie Rôle de L'Home et de L'Environment, Risques Sanitaires (J. P. Carmouze, M. Lucotte \& A. Boudou, org.), pp. 347-372, Paris: Collection Expertise Collégiale, Institut de Recherche pour Développement.

SANTOS, E. C. O.; JESUS, I. M.; BRABO, E. S.; LOUREIRO, E. C. B.; MASCARENHAS, A. F.; WEIRICH, J.; CÂMARA, V. M. \& CLEARY, D., 2000. Mercury exposure in riverside Amazon communities in Pará, Brazil. Environmental Research, 84:100-107.

SANTOS, E. C. O.; JESUS, I. M.; CÂMARA, V. M.; BRABO, E. S.; LOUREIRO, E. C. B.; MASCARENHAS, A. S.; WEIRICH, J.; LUIZ, R. R. \& CLEARY, D., 2001. Exposição ao Mercúrio em Índios Mundurukus da Comunidade de Sai Cinza, Estado do Pará, Brasil. Relatório Técnico. Belém: Seção de Meio Ambiente, Instituto Evandro Chagas. (mimeo.)

SANTOS, E. C. O.; LOUREIRO, E. C. B.; JESUS, I. M.; BRABO, E. S.; SILVA, R. S. U.; SOARES, M. C. P.; CÂMARA, V. M.; SOUZA, M. R. S. \& BRANCHES, F., 1995. Diagnóstico das condições de saúde de uma comunidade garimpeira na região do Tapajós, Itaituba, Pará, Brasil. Cadernos de Saúde Pública, 11:212-225.

VEIGA, M. M.; MEECH, J. A.; \& ONATE, N., 1994. Desforestation: A major source of mercury pollution in the Amazon. Nature, 368:816-817.

WHO (World Health Organization), 1990. Methylmercury. Environmental Health Criteria 101. Geneva: United Nations Environmental Program, International Program on chemical Safety, WHO.

Recebido em 13 de dezembro de 2001

Versão final reapresentada em 24 de maio de 2002 Aprovado em 14 de agosto de 2002 